\title{
Cancer Growth and Metastasis
}

\section{Bone Disease in Multiple Myeloma: Pathophysiology and Management}

\author{
Abdul Hameed ${ }^{1-3}$, Jennifer J. Brady ${ }^{4}$, Paul Dowling ${ }^{5}$, Martin Clynes $^{5}$ and Peter O'Gorman 5,6 \\ ${ }^{1}$ Medical Oncology Department, Shaukat Khanum Memorial Cancer Hospital and Research Centre, Lahore, Pakistan. ${ }^{2}$ Mater Misericordaie \\ University Hospital, Dublin, Ireland. ${ }^{3}$ Dublin City University, Dublin, Ireland. ${ }^{4}$ Department of Biochemistry, Mater Misericordaie University \\ Hospital, Dublin, Ireland. ${ }^{5}$ National Institute for cellular Biotechnology, Dublin City University, Dublin, Ireland. ${ }^{6}$ Hematology Department, \\ Mater Misericordaie University Hospital, Dublin, Ireland.
}

\begin{abstract}
Myeloma bone disease (MBD) is a devastating complication of multiple myeloma (MM). More than $80 \%$ of MM patients suffer from destructive bony lesions, leading to pain, fractures, mobility issues, and neurological deficits. MBD is not only a main cause of disability and morbidity in MM patients but also increases the cost of management. Bone destruction and lack of bone formation are main factors in the development of MBD. Some novel factors are found to be involved in the pathogenesis of $\mathrm{MBD}$, eg, receptor activator of nuclear factor kappa-B ligand (RANKL), osteoprotegerin (OPG) system (RANKL/OPG), Wingless (Wnt), dickkopf-1 (Wnt/DKK1) pathway. The addition of novel agents in the treatment of MM, use of bisphosphonates and other supportive modalities such as radiotherapy, vertebroplasty/kyphoplasty, and surgical interventions, all have significant roles in the treatment of MBD. This review provides an overview on the pathophysiology and management of MBD.
\end{abstract}

KEYWORDS: osteoclasts, osteoclastogenesis, osteoblasts, osteoblastogenesis, cytokines, bisphosphonates

\author{
CITATION: Hameed et al. Bone Disease in Multiple Myeloma: Pathophysiology and Management. Cancer Growth and Metastasis 2014:7 33-42 doi:10.4137/CGM.S16817. \\ RECEIVED: May 11, 2014. RESUBMITTED: June 18, 2014. ACCEPTED FOR PUBLICATION: June 20, 2014. \\ ACADEMIC EDITOR: Marc D. Basson, Editor in Chief \\ TYPE: Review \\ FUNDING: Authors disclose no funding sources \\ COMPETING INTERESTS: Authors disclose no potential conflicts of interest. \\ COPYRIGHT: $\odot$ the authors, publisher and licensee Libertas Academica Limited. This is an open-access article distributed under the terms of the Creative Commons \\ CC-BY-NC 3.0 License. \\ CORRESPONDENCE: hamiid34@gmail.com
}

This paper was subject to independent, expert peer review by a minimum of two blind peer reviewers. All editorial decisions were made by the independent academic editor. All authors have provided signed confirmation of their compliance with ethical and legal obligations including (but not limited to) use of any copyrighted material, compliance with ICMJE authorship and competing interests disclosure guidelines and, where applicable, compliance with legal and ethical guidelines on human and animal research participants.

\section{Introduction}

Multiple myeloma (MM) is a plasma cell disorder, characterized by bone marrow infiltration with clonal plasma cells, production of monoclonal immunoglobulin (paraprotein), and end organ damage including lytic lesions in the bones, renal impairment, hypercalcemia, and anemia. ${ }^{1}$

End organ damage is the main differentiating point of symptomatic from asymptomatic MM. In myeloma bone disease (MBD), lesions could be in the form of a classic discrete lytic lesion (radiolucent, plasmacytoma), widespread osteopenia, or multiple lytic lesions affecting any part of skeleton, preferably spine, skull, and long bones. ${ }^{2}$ The higher the number of lesions, the poorer the prognosis. ${ }^{3}$ Increased osteoclastogenesis with suppressed osteoblastic activity is the main mechanism of MBD. ${ }^{4}$ There are certain factors involved in stimulation and formation of osteoclasts (OCs) and reduction of osteoblastic activity. Recent advances in understanding of MBD showed that the receptor activator of nuclear factor kappa-B ligand (RANKL) and osteoprotegerin (OPG) system plays a key role in this regard. ${ }^{5}$

MBD on one hand results in increased disability, morbidity, and on the other hand leads to increased cost of treatment of these patients. ${ }^{6} \mathrm{MM}$ patients with bone disease not only need standard antimyeloma therapy but also require treatment with bisphosphonates (BPs), pain control, and a subgroup of patients may need radiotherapy and surgical interventions. ${ }^{7}$ This article focuses on different factors involved in the development of $\mathrm{MBD}$ and treatment modalities to manage this condition. 


\section{Normal Bone Remodeling}

Normal bone consists of a mineralized part and an organic part, made of collagen and noncollagen proteins. Bone remodeling is a continuous process, consisting of old bone resorption (osteoclastic activity) and new bone formation (osteoblastic activity). This process is well balanced in a normal person to keep the bones in healthy form. OCs and osteoblasts (OBs) are main types of cells involved in bone remodeling with the help of certain cytokines and hormones. ${ }^{8-10}$

Osteoclasts. OCs were first described in 1873. These are multinucleated cells originating from hematopoietic stem cells committed to monocyte-macrophage lineage. OCs originate not only from stem cells but also from mature monocyte-macrophage lineage when proper bone marrow microenvironment (BM-mic) is provided. ${ }^{11,12}$ OCs are the only cells known to cause bone resorption. They contain certain proteins, such as tartrate-resistant acid phosphatase (TRAP), tartrate-resistant trinucleotide phosphatase, carbonic anhydrase II, calcitonin receptors, and a few cathepsins (lysosomal proteases). ${ }^{13}$ The main function of OCs is bone resorption. The mechanism of bone degradation by OCs was not known until the 1980s when cathepsins were found to be the most important proteases involved in bone resorption. There are about seven cathepsins found in OCs (B, C, D, E, G, L, and K). Cathepsin-K is the most osteolytic, and inhibitors of cathepsin $\mathrm{K}$ are in trial to treat metastatic bone disease in cancer. ${ }^{14}$

Osteoblasts. OBs are mononuclear cells originating from mesenchymal stem cells (MSCs). They contain the enzyme alkaline phosphatase, which could be used as a marker of osteoblastic activity. ${ }^{11,15}$ Their normal location is near the bone surface where new bone is laid down. Their main function is bone formation, by collagen synthesis, osteocalcin (OCN) production, and mineralization. ${ }^{16,17} \mathrm{OBs}$ that become a part of mineralized matrix are called osteocytes, and they secrete the same type of biochemical agents as OBs. ${ }^{18,19}$

\section{Pathophysiology of Myeloma Bone Disease}

In contrast to normal bone remodeling, the coupling mechanism of $\mathrm{OCs}$ and $\mathrm{OBs}$ is lost in MM. Increased osteoclastic activity resulting in bone resorption and suppressed osteoblastic activity leading to decreased/absent bone formation are key factors in the development of $\mathrm{MBD}^{18}$ (Fig. 1). MBD is distinct from other metastatic diseases as there is no bone formation whereas in cancers like prostate and breast, the osteoclastic as well as osteoblastic activity is increased. ${ }^{20}$

Studies have shown that tissue hypoxia is a key factor in disease progression and metastasis of both solid tumors and hematopoietic malignancies. ${ }^{21}$ The expansion of plasma cells and neovascularization result in low oxygen in bone marrow niches. The resultant hypoxia in the BM-mic is considered as a triggering factor in myeloma cells (MCs) metastasis. ${ }^{22}$ This spread is mediated by the activation of epithelial to mesenchymal cell transition, resulting in a reduced adhesion in bone marrow and an increased spill of MCs into peripheral circulation. ${ }^{23}$

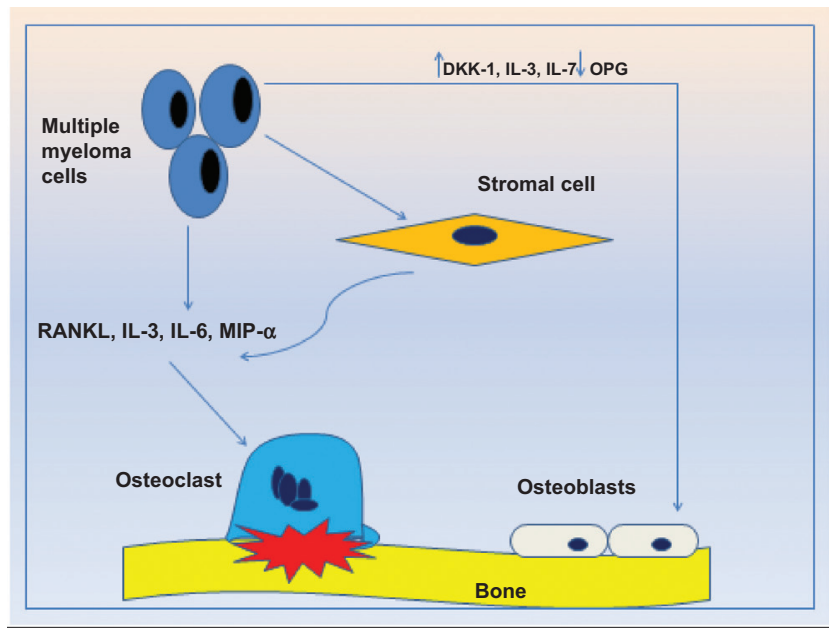

Figure 1. Pathophysiology of MBD. Interaction of MM cells with BMSCs leads to production of bone resorption factors, resulting in OC activation and increased bone resorption. On the other hand, MM cells inhibit the osteoblastic activity by production of inhibitory cytokines and reduced OPG production. ${ }^{20}$

\section{Increased osteoclastogenesis}

Increased osteoclastic activity is a major element of MBD, and an increase in bone resorption markers has been noted in patients with MM. ${ }^{4,24}$ There are many factors involved in formation and activation of OCs in myeloma patients. Interactions of MCs, T-lymphocytes, and bone marrow stromal cells (BMSCs) within the BM-mic contribute significantly in the development of MBD. The RANKL and OPG, a decoy receptor for RANKL, system plays the most important role in bone remodeling, and an imbalance in this system is one of the factors responsible for osteolysis seen in MM. ${ }^{25}$ Other chemokines are macrophage inflammatory peptide $1-\alpha$ (MIP-1 $1 \alpha)$, interleukin-3 (IL-3), interleukin-6 (IL-6), interleukin-7 (IL-7), stromal derived factor- $1 \alpha(\mathrm{SDF}-1 \alpha)$, and vascular endothelial growth factor (VEGF).

Bone Marrow microenvironment, MCs, T-lymphocytes, and BMSCs. The BM-mic includes both cellular and noncellular components. ${ }^{26}$ The cellular part consists of clonal plasma cells (MCs), immune cells (T-lymphocytes) and BMSCs, whereas proteins in extracellular matrix, cytokines, and growth factors in extracellular fluid make up the noncellular compartment. ${ }^{26} \mathrm{MCs}$ are noticed to be present in large amounts near the site of active bone resorption, and their interactions with BM-mic play an important role in the development and spread of disease. ${ }^{27,28} \mathrm{MCs}$ produce decoy receptor 3 (DcR3), which is responsible for OCs differentiation and activation. ${ }^{26} \mathrm{DcR} 3$, which belongs to tumor necrosis factor (TNF) receptor superfamily, is overexpressed in MCs and T-lymphocytes. ${ }^{29} \mathrm{MCs}$ not only secrete certain cytokines which are involved in MBD but also stimulate BMSCs and OBs to activate the RANKL/ OPG system which is a key factor in this process. ${ }^{18} \mathrm{MCs}$ can even interact with OCs and change themselves to multinucleated cells having bone resorptive properties. ${ }^{30}$ 
BMSCs have a critical role in the development of MBD. $\mathrm{MCs}$ adhere to BMSCs and this leads to the production of certain anti-apoptotic proteins and cytokines. ${ }^{31} \mathrm{MCs}$ and BMSCs interactions upregulate RANKL, IL-6, B-cell activating factor (BAFF), and activin A. ${ }^{32} \mathrm{BAFF}$, a member of TNF superfamily, is involved in the development of B-cells and promotes osteoclastogenesis and $\mathrm{MCs}$ survival..$^{32}$ Activin A belongs to the transforming growth factor (TGF) family, and high levels are noticed in advanced stage MM; furthermore, it acts as an activator and inhibitor of $\mathrm{OCs}$ and $\mathrm{OBs}$, respectively. ${ }^{33}$

Immune cells, particularly $\mathrm{T}$ lymphocytes, regulate $\mathrm{OC}$ and $\mathrm{OB}$ activity, survival, and function. ${ }^{26}$ In a study, Prabhala et al demonstrated a different subset of $\mathrm{T}$ helper cells (called Th17-1 cells). These cells secreted interleukin-17 (IL-17) in the bone marrow of MM patients. ${ }^{34}$ IL-17 not only mediates $\mathrm{MBD}$ but also increases $\mathrm{MC}$ survival. ${ }^{35}$ Activated T cells in MM produce osteoclastogenic cytokines such as IL-3, RANKL, DcR3, and TNF, resulting in enhanced osteolysis in these patients. ${ }^{29}$ Giuliani et al demonstrated that RANKL expression is upregulated in activated T-lymphocytes and CD3 positive cells in the bone marrow of myeloma patients with extensive bone disease. ${ }^{36,37}$ These findings are suggestive of T-lymphocytes involvement in the process of MBD.

RANKL/OPG System. RANKL, which belongs to TNF superfamily, is produced by BMSCs, OBs, and activated T-lymphocytes. MC interaction with BM-mic, hypercalcemia, and other bone resorpting agents such as parathyroid hormone (PTH), parathyroid hormone related protein (PTHrp), and vitamin $\mathrm{D} 3$ stimulate $\mathrm{BMSC}$ and $\mathrm{OBs}$ to increase production of RANKL. ${ }^{16,38}$

The number of precursor OCs is increased under the effect of macrophage-colony stimulating factor (M-CSF); then, RANKL through its RANK receptor on precursor OCs stimulates them to differentiate into mature OCs and reduces $\mathrm{OC}$ apoptosis resulting in increased osteoclastic activity. ${ }^{39}$ RANKL is a principal osteoclastogenic agent in MBD. Expression of RANKL is associated with a decrease in OPG levels in MM patients. MCs are not only the culprit for upregulating RANKL but at the same time they also internalize and degrade OPG, and consequently RANKL/OPG ratio is increased, which predicts poor survival in MM. ${ }^{40,41}$

Using immunohistochemistry stains, bone marrow biopsy specimens of myeloma patients were studied and an elevated RANKL with markedly reduced OPG expression was noted in BMSCs. This pattern was not observed in normal bone marrow or in other B-cell malignancies. ${ }^{42}$ Investigators also noticed that if RANKL is blocked with an inhibitor (eg, RANK.Fc) or OPG, it resulted in decreased disease burden and bone resorption. ${ }^{39}$ To sum up, RANKL/OPG imbalance is a major causative factor of $\mathrm{MBD}$, and could be a target to treat MBD.

Macrophage inflammatory protein-1 alpha (MIP-1 $\alpha$ ). MIP- $1 \alpha$ belongs to the $\mathrm{C}-\mathrm{C}$ chemokines family, and its main functions are cell adhesion and migration. It acts as a chemotactic factor for inflammatory cells (eg, monocytes, lymphocytes, dendritic cells, etc.). ${ }^{24}$ MIP- $1 \alpha$ is a strong osteoclastogenic factor and inhibits stem cells proliferation as well. It is produced by BMSCs, hematopoietic stem cells, and MCs. ${ }^{24,43}$

MIP- $1 \alpha$ plays an important role in the development of $\mathrm{MBD}$. It causes direct activation of $\mathrm{OCs}$, through chemokine receptors CCR1 and CCR5. MIP-1 $\alpha$ also acts on MCs (as they carry CCR1) enhancing their interactions with BMSCs and $\mathrm{OBs}$ to stimulate expression of RANKL and indirectly increasing bone resorption. ${ }^{24}$ Choi et al demonstrated high levels of MIP-1 $\alpha$ in whole bone marrow samples of myeloma patients; later on Shima et al reported an upregulation of this agent in purified plasma cells. ${ }^{44,45}$ Levels of MIP- $1 \alpha$ directly correlate with severity of bone disease and survival. In preclinical mouse models, blocking of MIP- $1 \alpha$ with antibody reduced severity of bone resorption. It seems that MIP- $1 \alpha$ is a major player in MBD and its downregulation may help in controlling MBD. ${ }^{46}$

Interleukin-6 (IL-6) and Interleukin-7 (IL-7). IL-6, produced by BMSCs, is involved in immune and inflammatory responses as well as in bone metabolism by activating certain pathways, like Ras/mitogen-activated protein (MAP), extracellular signal-regulated kinase (ERK) cascade, signal transducer and activator of transcription3 (STAT 3), and phosphoinositide 3-kinase/Akt cascade. Particularly, in myeloma, IL-6 increases survival of MCs and protects MCs from apoptosis. Its production is significantly increased in patients with active myeloma compared to monoclonal gammopathy of undetermined significance (MGUS) or smoldering myeloma. ${ }^{47,48}$ IL-6 enhances osteoclastic activity through increasing PTH effect, ${ }^{47}$ seemingly upregulates IL-7 activity ${ }^{26}$ to increase RANKL expression and bone resorption. ${ }^{36}$ High levels of IL-7 are found in peripheral blood and bone marrow of MM samples. ${ }^{49} \mathrm{In}$ vitro studies have shown a reduction in RANKL expression with anti-IL-7 and anti-IL-6 (mAb1339) antibodies. ${ }^{36,47,50}$

Interleukin-3 (IL-3). IL-3 is a cytokine secreted from activated lymphocytes. IL-3 level is increased in bone marrow plasma as well as in purified CD138 positive plasma cells of patients with MM. This substance along with MIP- $1 \alpha$ and RANKL significantly increases osteoclastic activity in MM, compared to MIP- $1 \alpha$ and RANKL alone. ${ }^{51}$ IL-3 had synergy with IL-6, leading to increased MCs growth. ${ }^{24}$

Osteopontin and VEGF. Osteopontin (OPN) is a noncollagenous protein present in bone, kidney, teeth, and epithelial lining tissues. It is produced by OBs and OCs and is involved in inflammatory responses, angiogenesis, apoptosis, and tumor metastasis. ${ }^{52}$ VEGF is a major factor in neovascularization and is produced by cells controlling angiogenesis, eg, vascular smooth muscle cells. There is increased production of VEGF and OPN by MCs and BMSCs; studies have shown a marked reduction in angiogenesis and osteoclastogenesis when VEGF and OPN were blocked. ${ }^{53,54}$ 
Stromal derived factor- 1 alpha (SDF-1 $\alpha$ ). SDF- $1 \alpha$ is produced by BMSCs and mediates its effect through CXCR4 receptor expressed on hematopoietic stem cells, lymphocytes, malignant cells and OC precursors. Its main role is hematopoietic stem cells homing, tumor proliferation, and migration.

MCs express CXCR4 and this expression is upregulated by certain factors, eg, VEGF. Thus, SDF- $1 \alpha$ increases homing, migration of $\mathrm{MCs}$, and enhances $\mathrm{OC}$ activity. Latter effect was much reduced with CXCR4 inhibitors, suggesting that $\mathrm{SDF}-1 \alpha$ plays a role in MBD. ${ }^{55}$

\section{Decreased osteoblastogenesis}

In $\mathrm{MBD}$, reduced bone formation secondary to decreased osteoblastic activity also plays a key role in severity of disease, resulting from extensive bone loss and no repair. ${ }^{56,57}$ Osteoblastic activity keeps pace with osteoclastic activity in the early phase of disease or when disease progresses from benign condition, like MGUS, and if patients continue to have high osteoblastic function, they do not develop MBD. ${ }^{58}$ There are certain factors involved in downregulation of osteoblastic activity, like Wnt/DKK1 pathway, IL-3, IL-7, secreted frizzled related protein-2 (sFRP-2), runt-related transcription factor 2 (Runx2), hepatocyte growth factor (HGF), and TGF- $\beta$.

Wingless (Wnt) signaling pathway. Wnt pathway has significant involvement in bone formation and remodeling. ${ }^{59}$ Wnt signaling pathway is implicated in various types of human cancers and could be a therapeutic target. ${ }^{60} \mathrm{Wnt}$ genes encode Wnt family glycoproteins, which transduce signals through
Frizzled family receptors with extracellular Wnt-binding and cytoplasmic Dishevelled-binding domains. ${ }^{61}$ These glycoproteins (Wnts) are involved in cell surface receptor activation, gene expression, cell proliferation, differentiation, and migration. ${ }^{62}$ Wnts also control embryogenesis, organ formation, development after birth, and regeneration of human tissues. ${ }^{63} \mathrm{Wnts}$ are classified as canonical - if they had capacity to inhibit phosphorylation of $\beta$-catenin and its degradationand noncanonical-if $\beta$-catenin levels do not change. ${ }^{64}$ $\beta$-Catenin is the major factor for OPG expression from OBs. ${ }^{59}$ It is demonstrated that inactivation of gene for LRP5 (lowdensity lipoprotein receptor-related protein 5), a Wnt signaling transducer, results in osteoporosis-pseudoglioma syndrome in humans, and gain-of-function mutations in LRP5 leads to a syndrome of hereditary high bone density. ${ }^{65,66}$ These findings suggest that activation of this pathway causes increased osteoblastic activity and inhibition will reduce bone formation (Fig. 2) ${ }^{67}$ This pathway is regulated by two main antagonists, DKK1 and sFRP-2.

Dickkopf-1 (DKK1). DKK1 is expressed by OBs and BMSCs, it antagonizes the Wnt pathway resulting in inhibition of OBs maturation and new bone formation. ${ }^{68}$ Initially overexpression of DKK1 in bone marrow aspirate of myeloma patients was noted by cDNA microarray. Its concentration was increased in bone marrow plasma and peripheral blood of MM patients compared to MGUS or healthy controls.

As a result of high expression of DKK1, MSCs do not differentiate to OBs. ${ }^{69} \mathrm{In}$ in vitro studies it has been noticed

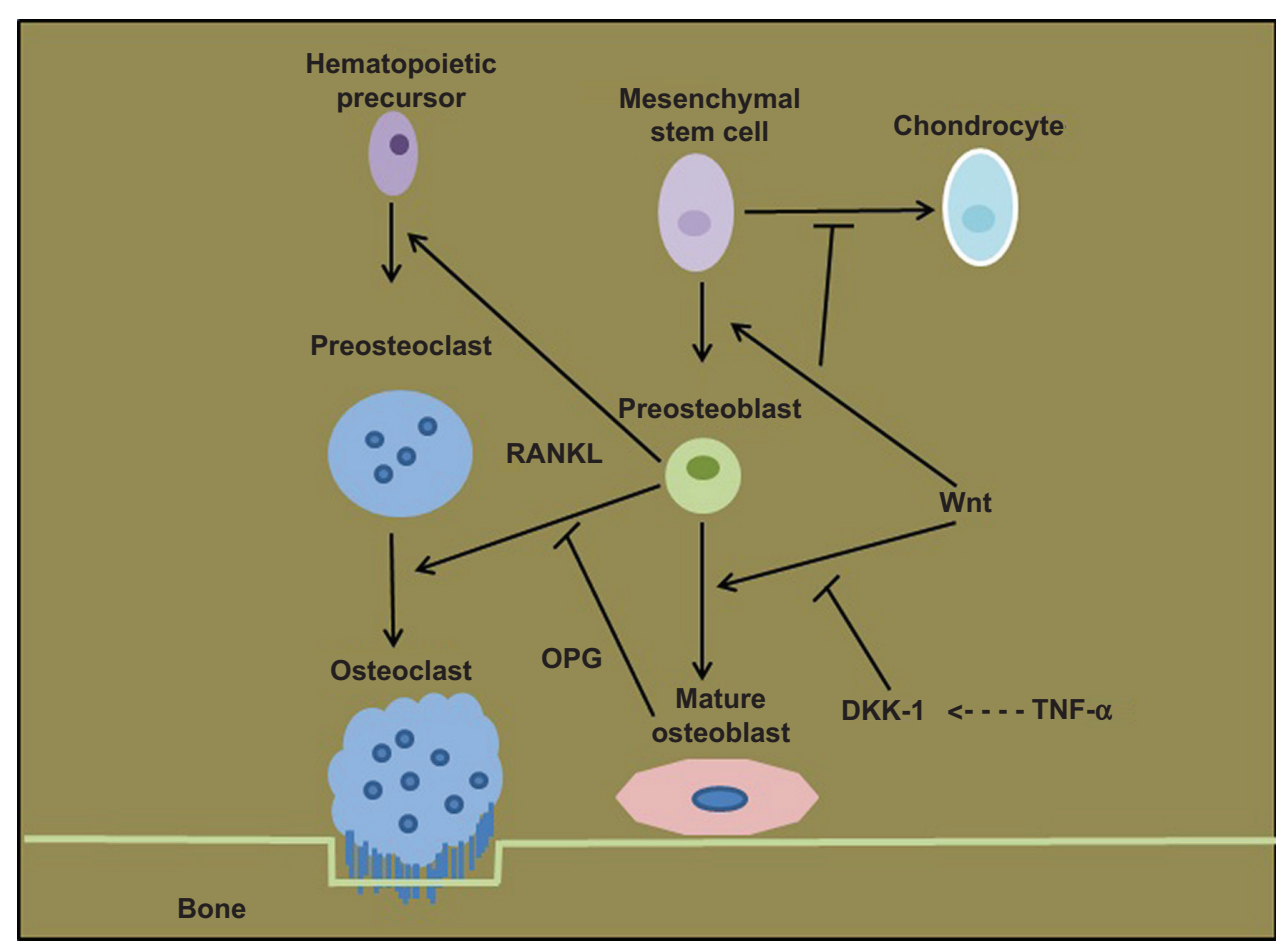

Figure 2. Wnt signaling pathway. Wnt signaling stimulates OB differentiation from MSCs. Blockade of this pathway through DKK1 inhibits OB formation. Wnt signaling upregulates RANKL expression from OB precursors resulting in increased osteoclastic activity and bone resorption. Activation of Wnt pathway increases OPG production from OB which in turn downregulates RANKL-driven osteoclastogenesis. ${ }^{67}$ 
that DKK1 also increased IL-6 expression from MSCs, contributing to MCs survival and growth. Ya-Wei Qiang et al demonstrated that blockade of Wnt signaling also reduces OPG, and an increase in RANKL expression from DKK1 affected MSCs and by this adding to osteoclastogenesis. ${ }^{68,70}$ In a mouse model, anti-DKK1 (BHQ880) antibody reduced MBD, increased osteoblastic activity, and a marked reduction in disease burden has been reported. ${ }^{71,72}$

Secreted frizzled related protein-2 (sFRP-2). sFRP-2 is another Wnt pathway antagonist, and it inhibits binding of Wnt to membrane bound receptor, frizzled, resulting in downregulation of osteoblastic activity. ${ }^{56}$ There are reports that it is expressed by MCs, and recombinant sFRP-2 inhibited differentiation of the murine OB-like cell lines. ${ }^{73}$ These observations suggest that this sFRP-2 may have a role in MBD.

$I L-3$. IL-3 plays a dual role in bone remodeling by OC stimulation and indirectly $\mathrm{OB}$ inhibition. Ehrlich et al demonstrated that IL-3 inhibited basal and bone morphogenic protein 2 (BMP-2), which stimulates OBs formation in a dose-dependent manner both in mouse and human BMSCs. Marrow plasma from MM patients with high IL-3 levels inhibited OBs activity which could be blocked with anti-IL-3. This cytokine increased the number of CD45 positive hematopoietic cells in stromal cell cultures, and depletion of CD45 positive cells resulted in downregulation of IL-3 inhibitory effects on OBs. ${ }^{74}$

Runt-related transcription factor 2 (Runx2)/corebinding factor runt domain alpha subunit 1 (CBFA1). Runx2/CBFA1 plays an important role in formation and differentiation of OBs from MSCs and BMSCs. Activation of Runx2/CBFA1 in human BMSCs and preosteoblastic cells induces high expression of osteoblastic markers like alkaline phosphatase and OCN. Moreover, mice that are Runx2/ CBFA1 negative lack osteoblastic activity and bone formation. ${ }^{75}$ When human MCs were cocultured with preosteoblastic cells, an inhibitory effect on $\mathrm{OB}$ formation was noted along with reduced activity of Runx2/CBFA1. In the same study a markedly reduced number of Runx2/CBFA1 positive cells were noted in bone marrow biopsy specimens of myeloma patients who have osteolytic lesions compared to who do not have MBD. ${ }^{76,77}$

$I \boldsymbol{L}-7$. IL-7 has a dual affect, increasing osteoclastic activity as well as inhibiting $\mathrm{OB}$ stimulation and maturation. High levels of IL-7 had been demonstrated in bone marrow of MM patients, and it inhibited both early and late OBs differentiation and formation. IL-7 also decreased Runx2/CBFA1 activity. 75,78

Transforming growth factor- $\boldsymbol{\beta}$ (TGF- $\beta$ ). TGF- $\beta$ is a growth factor released from bone matrix during bone resorption and acts to inhibit terminal osteoblastic differentiation. It has been demonstrated in vitro studies that blocking of TGF- $\beta$ leads to inhibition of MCs growth as well as increased differentiation and formation of OBs. ${ }^{54,79}$

Hepatocyte growth factor (HGF). HGF is produced by $\mathrm{MCs}$, and an elevated level had been demonstrated in the serum of patients suffering from MM compared to healthy individuals. In addition, high levels were associated with poor survival. Increased level of HGF negatively affects anabolic markers, through inhibition of BMP-2 and Runx2/CBFA1. ${ }^{52}$

\section{Management of Myeloma Bone Disease}

About $80-90 \%$ of myeloma patients suffer from osteolytic lesions during the course of disease affecting axial and appendicular skeleton. As a result of $\mathrm{MBD}$, patients may suffer from bone pain (70-80\%), fractures (50-60\%), hypercalcemia (15\%), spinal cord compression (2-3\%), decreased quality of life, and poor mobility. ${ }^{80}$

Management of MBD needs a multidisciplinary approach including antimyeloma treatment and the use of other supportive modalities like BPs, radiotherapy, pain control, vertebro-kyphoplasty, and surgical interventions.

Systemic antimyeloma treatment. Disease control is a major element in treatment of MBD. Patients should be started on a standard antimyeloma therapy. The choice of systemic antimyeloma treatment depends upon patient age, suitability for high dose therapy/autologous stem cell transplant, comorbidities, availability of certain drugs, and patient choices. More detail in this context is beyond the scope of this article.

Bisphosphonates (BPs). Bisphosphonate therapy (BPT) is standard of care in treatment of MBD. Derivatives of pyrophosphates and nitrogen-containing $\mathrm{BPs}$ (eg, pamidronate, zoledronic acid) are 10 to 10,000 times more potent than nonnitrogen containing BPs. Their main function is inhibition of OCs maturation and formation, enhancement of OCs apoptosis, and reduction of their adherence to bone by inhibition of farnesyl pyrophosphate synthase. In MM, intravenous agents are preferably used, as oral forms are less effective. ${ }^{81}$

Pamidronate and zoledronic acid are widely used nitrogen-containing BPs. They reduce pain and other skeletal related events (SREs), eg, vertebral fractures, hypercalcemia, and are equally effective in this regard. They are recommended in symptomatic MM, with or without evidence of bone disease. ${ }^{82} \mathrm{~A}$ placebo-controlled trial with intravenous pamidronate revealed significant decrease in SRE, better pain control, and improved quality of life in pamidronate group. ${ }^{83}$ In the Medical Research Council (MRC) Myeloma IX trial, zoledronic acid has been shown to have antimyeloma effect and was found to be better than sodium clodronate in reduction of SREs, progression free survival (PFS), and overall survival (OS) ${ }^{84}$ Pamidronate $90 \mathrm{mg}$ intravenous infusion is given at least in two hours, zoledronic acid dose $4 \mathrm{mg}$ is given in 15 minutes and to be repeated every three to four weeks. Dose adjustment is required for zoledronic acid if patient has renal impairment. Pamidronate can be used in patients with severe renal impairment with increased infusion duration of two to four hours and reduced dose of $30 \mathrm{mg} .{ }^{82}$

Patients on long-term BPT should be monitored for adverse effects like renal toxicity and osteonecrosis of jaw 
(ONJ). Pamidronate-associated renal damage may present as proteinuria or nephrotic syndrome, and zoledronic acid may cause acute tubular necrosis, resulting in renal impairment. Serum creatinine and urinary protein should be monitored regularly in these patients. ${ }^{85}$ Another serious adverse outcome with BPT is ONJ with incidence of 1-10\% in long-term users of these agents. Incidence is higher with zoledronic acid as compared to pamidronate ( $10 \%$ vs. $4 \%) .{ }^{86} \mathrm{ONJ}$ is defined as presence of exposed bone in the mandible or maxilla in patients receiving BPT which does not heal within eight weeks in spite of proper dental management. Some patients may have pain or fistulae and a few may be asymptomatic. There are certain risk factors for this complication including tooth extraction or other invasive dental surgery, longer duration of BPT, poor oral hygiene, older age, and longer survival. ${ }^{87}$

All patients going to start BPT should have dental review and if any invasive dental procedures needed it should be done before starting treatment. After invasive dental procedures BPT may be started 90 days post-procedures, seemingly if patient needed dental surgery which is inevitable, then it should be done 90 days after last infusion of BPs.

Optimal duration of BPT is still an open debate; however, with risks of ONJ and other adverse events with their long-term use, there is a concern among clinicians regarding indefinite use of these agents. Attal et al reported no significant difference in occurrence of SREs in patients who were on maintenance pamidronate (21\% and $18 \%$ ) compared to no maintenance group (24\%) at 29 months of follow up. ${ }^{88}$

International Myeloma Working Group (IMWG) suggested stopping BPT after one year in patients who are in complete remission (CR) or in very good partial remission (VGPR) and no active bone disease. Patients with less than VGPR, and ongoing bone disease should have BPT for up to 2 years. After two years, BPT can be stopped in patients with no active bone disease. If bony disease persists, further use of $\mathrm{BPs}$ is at the discretion of treating physician. Pamidronate or clodronate is preferred for long-term use ( $>2$ years). ${ }^{89}$ Based on the above statement and available data, in certain groups of myeloma patients, BPT could be stopped after two years of treatment; however, remission status, extent of bone disease, and patient preference should be taken into account before making this decision.

Pain control. Majority of myeloma patients (70-80\%) suffer from pain, and this could be the major complaint at the time of diagnosis. Pain in MM can be caused by disease process and bone resorption, in some cases plasmacytoma may cause nerve root compression. During the course of disease, patients may have pain as side effects of treatments, eg, thalidomide- or bortezomib-related peripheral neuropathy. An assessment should be made about pain severity, using pain score based on an imaginary pain scale from 0 to 10 (10 would be worst pain). Score of 5 or above would be moderate to severe pain, and patients who persistently have this reading should be referred to pain management team. ${ }^{90}$ For mild pain paracetamol can be used,
NSAIDS should be avoided if possible because of risk of renal toxicity. Patients who have moderate to severe pain may benefit from opioids like tramadol, oxycodone, fentanyl patches, and morphine. Patients should be observed for side effects like sedation and constipation with the use of opioids analgesia, particularly in elderly. BPs are also helpful in pain control. In cases where pain does not settle with pharmacological treatment, other modalities like radiotherapy, vertebroplasty/kyphoplasty and even orthopedic interventions may be required. ${ }^{1}$

Radiotherapy. Historically, radiotherapy has been an important part of MM treatment. Radiotherapy is a treatment of choice in solitary bone plasmacytoma and could be curative in some cases. Other indications are as pain control measure, spinal cord compression, pathological fractures, soft tissue plasmacytomas, and control of local neurological symptoms. Major risk of radiotherapy is permanent bone marrow damage, which can affect stem cell harvest in future. Most common indication is pain control where a success rate of more than $90 \%$ has been noted. ${ }^{92}$

Vertebroplasty/kyphoplasty. This is a minimal invasive procedure, carried out under local or general anesthesia. Vertebroplasty includes fluoroscopic percutaneous injection of polymethylmethacrylate, bone cement, into vertebral body for stabilization or pain relief. Kyphoplasty is the same type of procedure where inflatable balloon is placed into the vertebral body to expand it and then followed by injection of bone cement. Potential complication of this procedure is cement leakage which could cause nerve irritation or cord compression and embolization of cement.

Both procedures are effective and safe in reduction of pain and improvement of mobility in patients with compression fractures of vertebrae. ${ }^{93}$ In one study, 26 patients with painful lesions because of MM had vertebroplasty. $77 \%$ of these patients had pain reduction after procedure, $1 / 3$ of these became opioids independent, and $80 \%$ had improvement in mobility. There were three patients who had small cement leaks at the time of procedure, symptoms were resolved within 24 hours and there was no cement or fat emboli, bleeding, or infections. ${ }^{94}$ These procedures are less invasive with excellent outcome in improvement of pain and mobility.

Surgical intervention. Surgery has a role in MM patients with fractures of long bones. Other indications are intractable pain not resolving after nonsurgical measures, spinal instability manifested as fracture or neurological deficit, and cord compression with epidural tumor. However, hematologist and radiation oncologist opinions should be sought before deciding on surgery.

\section{Novel Agents in the Treatment of MBD}

Anti-RANKL. RANKL is a key factor in pathogenesis of MBD. Denosumab (AMG 126) is a fully humanized antibody to RANKL; it has high affinity for RANKL leading to inhibition of RANKL-RANK interaction resulting in suppression of bone resorption. Body et al demonstrated efficacy and safety 
of denosumab in patients with breast cancer and MM with bone lesions; patients were given single dose of denosumab or pamidronate. Antiresorptive effects were assessed by changes in urine and serum level of $\mathrm{N}$-telopeptide (a bone resorption marker). Results showed significant reduction in bone resorption marker with single dose of denosumab, which was similar to pamidronate but more sustained. ${ }^{95}$ In another phase III trial of denosumab compared with zoledronic acid in patients with solid tumors metastatic to bone (excluding breast and prostate) and patients with MM, it was noticed that denosumab was not superior to zoledronic acid in decreasing SREs. ${ }^{96}$ This drug is not approved for MBD at this point.

Proteosome inhibitor (bortezomib). The proteosome pathway plays a role in $\mathrm{OB}$ differentiation and its inhibition can induce new bone formation in rodents by increasing expression of BMP2, a potent inducer of $\mathrm{OB}$ differentiation. ${ }^{97}$ Bortezomib is a backbone of MM treatment these days. Recent clinical trials have shown that bortezomib may increase osteoblastic activity as well. In one study, osteoblastic markers (bone alkaline phosphatase, BAP, and OCN) in serum of MM patients treated with bortezomib alone or in combination with dexamethasone were examined. The control group was MM patients treated with non-bortezomib based regimens. Quantification was made before treatment and three months after treatment. There was significant rise in BAP and OCN in the bortezomib group. The rise in BAP and OCN in control group was not statistically significant. ${ }^{98}$ In another study, the bortezomib significantly increased transcription factor Runx2/CBFA1 activity in human OBs and also increased the number of OBs. ${ }^{78}$ Bortezomib not only increased osteoblastogenesis but also demonstrated anti-osteoclastic activity in different studies. ${ }^{99,100}$

Immunomodulatory drugs. Immunomodulatory drugs (IMiDs) are thalidomide analogues, with significant antimyeloma activity. ${ }^{101} \mathrm{IMiDs}$ induce MCs apoptosis and enhance antimyeloma $\mathrm{T}$ cell and natural killer cell immunity. ${ }^{102}$ These agents also inhibit angiogenesis, leading to BM-mic alterations and a decrease in MCs growth and survival. ${ }^{103}$ In one study, thalidomide derivative CC-4047 inhibited osteoclastogenesis through downregulation of PU.1-a key factor for hematopoietic differentiation. ${ }^{104}$ In another study, a reduction in RANKL/OPG ratio and bone osteolysis was noticed in $\mathrm{MM}$ patients treated with thalidomide-based regimen; however, no change was seen in bone formation markers. ${ }^{105}$ Recently, a combination of lenalidomide with high dose dexamethasone has shown only marginal decline in bone resorption markers (only in those patients who responded to therapy); ${ }^{106}$ however, when lenalidomide was combined with bortezomib (VRD-bortezomib, lenalidomide, dexamethasone), a significant reduction and increment was seen in bone resorption and formation markers, respectively. ${ }^{106}$

Anti-DKK1. Reduced OB function is also an important element of MBD. High expression of DKK1 has been found in MM patients with extensive bone disease. DKK1 not only has direct inhibitory effect on $\mathrm{OB}$ but also disrupts Wnt signaling, resulting in increased OC activation. ${ }^{107}$ Yaccoby et al reported (study in SCID-rab mice) that antiDKK1 stimulated OB activity, reduced osteoclastogenesis, and promoted bone formation in myelomatous and nonmyelomatous bones. The bone anabolic effect of anti-DKK1 was associated with reduced MM burden. ${ }^{71}$ Based on these observations, anti-DKK1 may be a potential treatment agent in MBD in coming years.

OPG agonists. OPG is a decoy receptor for RANKL, and it blocks differentiation and activation of OCs. In one study, the safety and efficacy of recombinant OPG (AMGN 0007) was evaluated. Patients with MM and breast cancer with radiologically confirmed bony lesions were given single dose of AMGN 0007 or pamidronate $90 \mathrm{mg}$. Its effects were assessed by measuring resorption marker NTX. AMGN 0007 caused a decrease in the level of NTX and was comparable to pamidronate treated patients, and was well tolerated. OPG agonists are in very early stages of trials and may need a few more years before their role in MBD is established. ${ }^{108}$

Bruton tyrosine kinase inhibitors. Bruton tyrosine kinase (BTK) is a nonreceptor tyrosine kinase, expressed in many hematopoietic cells and carries a significant importance in B-lymphocytes development and function. ${ }^{109}$ The BTK pathway is a potential therapeutic target in a variety of B-cell malignancies. ${ }^{110}$ PCI-32765 (ibrutinib), the first BTK inhibitor, has shown significant antitumor activity in B-cell lymphoproliferative disorders such as chronic lymphocytic leukemia and mantle cell lymphoma. ${ }^{111,112}$ Recent studies suggested strong BTK expression in $\mathrm{MCs}^{113}$ and involvement of this pathway in the development of MBD. ${ }^{114} \mathrm{BTK}$ is selectively expressed in OCs but not in OBs. ${ }^{115}$ Investigators have demonstrated that BTK inhibitors (eg, PCI-32765 and LFM-A13) not only reduced tumor burden in MM but also decreased osteoclastogenesis and bone osteolysis. ${ }^{113,114}$ More studies are needed to establish the role of BTK inhibitors in the management of MBD. Table 1 provides a summary of novel therapeutic agents for MBD.

\section{Summary}

The majority of MM patients suffer from MBD, and this is a main cause of morbidity and disability in this disease. Increased osteoclastic activity and reduced osteoblastic function are key factors in MBD pathogenesis, which is orchestrated by different cytokines/chemokines, eg, RANKL/OPG, Wnt/DKK1, IL-6, IL-7, IL-3, MIP, and many others which are produced by $\mathrm{MCs}, \mathrm{BMSC}$, and $\mathrm{OBs}$. BPs are the mainstay of treatment in MBD; however, radiotherapy, vertebrokyphoplasty, and other surgical procedures have a role in a subgroup of patients.

Osteolysis is an independent risk factor for OS in symptomatic MM patients. In this era of targeted therapies, better understanding of pathogenesis of $\mathrm{MBD}$ has resulted 
Table 1. Novel agents for MBD.

\begin{tabular}{|llll|}
\hline DRUGS & ACTIONS & TARGET CELLS & REFERENCE \\
\hline Bisphosphonates & Osteoclast inhibitor & OCs & 90 \\
\hline Denosumab & RANKL-antagonist & OCs & 100 \\
\hline Bortezomib & $\begin{array}{l}\text { Osteoblast stimulator } \\
\text { Osteoclast inhibitor }\end{array}$ & OBs, OCs & 103,105 \\
\hline IMiDs & $\begin{array}{l}\text { PU.1 down regulation } \\
\text { Osteoclasts inhibitor }\end{array}$ & OCs & 110,112 \\
\hline BHQ880 & DKK-1-antagonist & OBs & 76 \\
\hline AMG 0007 & OPG-agonist & OCs & 114 \\
\hline PCI-32765 & BTK-inhibitor & OCs & 119 \\
\hline LFM-A13 & BTK-inhibitor & OCs & 120 \\
\hline SB431542 and Ki26894 & TGF- $\beta$ inhibitor & OBs & 83 \\
\hline T140 & CXCR4-inhibitor & OCs & 122 \\
\hline mAb-1339 & Anti-IL6 & OCs & 54 \\
\hline
\end{tabular}

in recognition of new targets in this disease. Inhibitors of RANKL, DKK1, IL-3, 1L-6, MIP, and BTK in combination with novel antimyeloma agents could be promising options in future. It is well documented that lytic lesions in MM never heal; however, novel therapeutic agents like bortezomib not only have antimyeloma effect but also increases osteoblastic activity resulting in the recovery of lytic lesions. With the addition of novel agents in the treatment of MM, median survival has significantly increased. This demands that the skeletal complications should be managed more aggressively to improve the quality of life. With ongoing clinical trials it is anticipated that a wide range of targeted therapies may become available in future to treat MBD more effectively.

\section{Author Contributions}

Conceived the concept: AH, PO. Analyzed the data: AH, PO, JB, PD, MC. Wrote the first draft of the manuscript: AH. Contributed to the writing of the manuscript: PO, JB, MC. Agree with manuscript results and conclusions: $\mathrm{AH}, \mathrm{PO}, \mathrm{JB}, \mathrm{MC}, \mathrm{PD}$. Jointly developed the structure and arguments for the paper: AH, PO, JB, MC, PD. Made critical revisions and approved final version: $\mathrm{AH}, \mathrm{PO}, \mathrm{JB}$, MC, PD. All authors reviewed and approved of the final manuscript.

\section{REFERENCES}

1. Kyle RA, Rajkumar SV. Criteria for diagnosis, staging, risk stratification and response assessment of multiple myeloma. Leukemia. 2009;23(1):3-9.

2. Kyle RA, Therneau TM, Rajkumar SV, Larson DR, Plevak MF, Melton LJ. Incidence of multiple myeloma in Olmsted County, Minnesota. Cancer. 2004; 101(11):2667-2674.

3. Walker R, Barlogie B, Haessler J, et al. Magnetic resonance imaging in multiple myeloma: diagnostic and clinical implications. J Clin Oncol. 2007;25(9): 1121-1128.

4. Bataille R, Chappard D, Marcelli C, et al. Mechanisms of bone destruction in multiple myeloma: the importance of an unbalanced process in determining the severity of lytic bone disease. J Clin Oncol. 1989;7(12):1909-1914.
5. Vanderkerken K, De Leenheer E, Shipman C, et al. Recombinant osteoprotegerin decreases tumor burden and increases survival in a murine model of multiple myeloma. Cancer Res. 2003;63(2):287-289.

6. Bruce NJ, McCloskey EV, Kanis JA, Guest JF. Economic impact of using clodronate in the management of patients with multiple myeloma. BrJ Haematol. 1999;104(2):358-364.

7. Adamietz IA, Schober C, Schulte RW, Peest D, Renner K. Palliative radiotherapy in plasma cell myeloma. Radiother Oncol. 1991;20(2):111-116.

8. Millett PJ. Synthetic function and regulation of osteoblasts: current knowledge and applications. MJM. 1995;1:138-146.

9. Phill PA. Bone remodelling. Br J Orthod. 1998;25:101-107.

10. Papadopoulou EC, Batzios SP, Dimitriadou M, Perifanis V, Garipidou V. Multiple myeloma and bone disease: pathogenesis and current therapeutic approaches. Hippokratia. 2010;14(2):76-81.

11. Udagawa N, Takahashi N, Akatsu T, et al. Origin of osteoclasts: mature monocytes and macrophages are capable of differentiating into osteoclasts under a suitable microenvironment prepared by bone marrow-derived stromal cells. Proc Natl Acad Sci U S A. 1990;87(18):7260-7264.

12. Bar-Shavit $Z$. The osteoclast: a multinucleated, hematopoietic-origin, boneresorbing osteoimmune cell. J Cell Biochem. 2007;102(5):1130-1139.

13. Janckila AJ, Takahashi K, Sun SZ, Yam LT. Tartrate-resistant acid phosphatase isoform 5 b as serum marker for osteoclastic activity. Clin Chem. 2001;47(1):74-80.

14. Delaisse JM, Eeckhout Y, Vaes G. Inhibition of bone resorption in culture by inhibitors of thiol proteinases. Biochem J. 1980;192(1):365-368.

15. George J, Kuboki Y, Miyata T. Differentiation of mesenchymal stem cells into osteoblasts on honeycomb collagen scaffolds. Biotechnol Bioeng. 2006;95(3): 404-411.

16. McSheehy PM, Chambers TJ. Osteoblast-like cells in the presence of parathyroid hormone release soluble factor that stimulates osteoclastic bone resorption. Endocrinology. 1986;119(4):1654-1659.

17. Owen TA, Aronow MS, Barone LM, Bettencourt B, Stein GS, Lian JB. Pleiotropic effects of vitamin $\mathrm{D}$ on osteoblast gene expression are related to the proliferative and differentiated state of the bone cell phenotype: dependency upon basal levels of gene expression, duration of exposure, and bone matrix competency in normal rat osteoblast cultures. Endocrinology. 1991;128(3): 1496-1504.

18. Giuliani N, Rizzoli V, Roodman GD. Multiple myeloma bone disease: pathophysiology of osteoblast inhibition. Blood. 2006;108(13):3992-3996.

19. Holtrop ME. Light and electron microscopic structure of bone forming cells. The osteocyte. Bone. 1990;1:1.

20. Roodman GD. Pathogenesis of myeloma bone disease. Blood Cells Mol Dis. 2004;32(2):290-292.

21. Zhang Y, Li M, Yao Q Chen C. Recent advances in tumor hypoxia: tumor progression, molecular mechanisms, and therapeutic implications. Med Sci Monit. 2007;13(10):RA175-RA180.

22. Shain K. Metastatic myeloma? Blood. 2012;119(24):5612-5613.

23. Puisieux A, Brabletz T, Caramel J. Oncogenic roles of EMT-inducing transcription factors. Nat Cell Biol. 2014;16(6):488-494.

24. Terpos E, Dimopoulos MA. Myeloma bone disease: pathophysiology and management. Ann Oncol. 2005;16(8):1223-1231. 
25. Giuliani N, Colla S, Rizzoli V. New insight in the mechanism of osteoclast activation and formation in multiple myeloma: focus on the receptor activator of NF-kappaB ligand (RANKL). Exp Hematol. 2004;32(8):685-691.

26. Oranger A, Carbone C, Izzo M, Grano M. Cellular mechanisms of multiple myeloma bone disease. Clin Dev Immunol. 2013;2013:289458.

27. Andrews SW, Kabrah S, May JE, Donaldson C, Morse HR. Multiple myeloma: the bone marrow microenvironment and its relation to treatment. BrJ Biomed Sci. 2013;70(3):110-120.

28. Valentin-Opran A, Charhon SA, Meunier PJ, Edouard CM, Arlot ME. Quantitative histology of myeloma-induced bone changes. Br J Haematol. 1982;52(4): 601-610.

29. Colucci S, Brunetti G, Mori G, et al. Soluble decoy receptor 3 modulates the survival and formation of osteoclasts from multiple myeloma bone disease patients. Leukemia. 2009;23(11):2139-2146.

30. Calvani N, Cafforio P, Silvestris F, Dammacco F. Functional osteoclast-like transformation of cultured human myeloma cell lines. Br JHaematol. 2005;130(6): 926-938.

31. Hideshima T, Bergsagel PL, Kuehl WM, Anderson KC. Advances in biology of multiple myeloma: clinical applications. Blood. 2004;104(3):607-618.

32. Tai YT, Li XF, Breitkreutz I, et al. Role of B-cell-activating factor in adhesion and growth of human multiple myeloma cells in the bone marrow microenvironment. Cancer Res. 2006;66(13):6675-6682.

33. Terpos E, Kastritis E, Christoulas D, et al. Circulating activin-A is elevated in patients with advanced multiple myeloma and correlates with extensive bone involvement and inferior survival; no alterations post-lenalidomide and dexamethasone therapy. Ann Oncol. 2012;23(10):2681-2686.

34. Prabhala RH, Pelluru D, Fulciniti M, et al. Elevated IL-17 produced by TH17 cells promotes myeloma cell growth and inhibits immune function in multiple myeloma. Blood. 2010;115(26):5385-5392.

35. Noonan K, Marchionni L, Anderson J, Pardoll D, Roodman GD, Borrello I. A novel role of IL-17-producing lymphocytes in mediating lytic bone disease in multiple myeloma. Blood. 2010;116(18):3554-3563.

36. Giuliani N, Colla S, Sala R, et al. Human myeloma cells stimulate the receptor activator of nuclear factor-kappa B ligand (RANKL) in T lymphocytes: a potential role in multiple myeloma bone disease. Blood. 2002;100(13):4615-4621.

37. Nosaka K, Miyamoto T, Sakai T, Mitsuya H, Suda T, Matsuoka M. Mechanism of hypercalcemia in adult T-cell leukemia: overexpression of receptor activator of nuclear factor kappaB ligand on adult T-cell leukemia cells. Blood. 2002; 99(2):634-640

38. Feige U. Osteoprotegerin. Ann Rheum Dis. 2001;60(suppl 3):iii81-iii84.

39. Yaccoby S, Pearse RN, Johnson CL, Barlogie B, Choi Y, Epstein J. Myeloma interacts with the bone marrow microenvironment to induce osteoclastogenesis and is dependent on osteoclast activity. Br J Haematol. 2002;116(2):278-290.

40. Terpos E, Szydlo R, Apperley JF, et al. Soluble receptor activator of nuclear factor kappaB ligand-osteoprotegerin ratio predicts survival in multiple myeloma: proposal for a novel prognostic index. Blood. 2003;102(3):1064-1069.

41. Sezer O, Heider U, Zavrski I, Kuhne CA, Hofbauer LC. RANK ligand and osteoprotegerin in myeloma bone disease. Blood. 2003;101(6):2094-2098.

42. Pearse RN, Sordillo EM, Yaccoby S, et al. Multiple myeloma disrupts the TRANCE/osteoprotegerin cytokine axis to trigger bone destruction and promote tumor progression. Proc Natl Acad Sci U S A. 2001;98(20):11581-11586.

43. Watanabe T, Kukita T, Kukita A, et al. Direct stimulation of osteoclastogenesis by MIP-1alpha: evidence obtained from studies using RAW264 cell clone highly responsive to RANKL. J Endocrinol. 2004;180(1):193-201.

44. Choi SJ, Oba Y, Gazitt Y, et al. Antisense inhibition of macrophage inflammatory protein 1-alpha blocks bone destruction in a model of myeloma bone disease. J Clin Invest. 2001;108(12):1833-1841.

45. Uneda S, Hata H, Matsuno F, et al. Macrophage inflammatory protein-1 alpha is produced by human multiple myeloma (MM) cells and its expression correlates with bone lesions in patients with MM. Br J Haematol. 2003;120(1):53-55.

46. Oyajobi BO, Franchin G, Williams PJ, et al. Dual effects of macrophage inflammatory protein-1alpha on osteolysis and tumor burden in the murine 5TGM1 model of myeloma bone disease. Blood. 2003;102(1):311-319.

47. de la Mata J, Uy HL, Guise TA, et al. Interleukin-6 enhances hypercalcemia and bone resorption mediated by parathyroid hormone-related protein in vivo. J Clin Invest. 1995;95(6):2846-2852.

48. Bataille R, Chappard D, Klein B. The critical role of interleukin-6, interleukin$1 \mathrm{~B}$ and macrophage colony-stimulating factor in the pathogenesis of bone lesions in multiple myeloma. Int J Clin Lab Res. 1992;21(4):283-287.

49. Silbermann R, Roodman GD. Myeloma bone disease: pathophysiology and management. J Bone Oncol. 2013;2(2):59-69.

50. Fulciniti M, Hideshima T, Vermot-Desroches C, et al. A high-affinity fully human anti-IL-6 mAb, 1339, for the treatment of multiple myeloma. Clin Cancer Res. 2009;15(23):7144-7152.

51. Lee JW, Chung HY, Ehrlich LA, et al. IL-3 expression by myeloma cells increases both osteoclast formation and growth of myeloma cells. Blood. 2004; 103(6):2308-2315.
52. Standal T, Hjorth-Hansen H, Rasmussen T, et al. Osteopontin is an adhesive factor for myeloma cells and is found in increased levels in plasma from patients with multiple myeloma. Haematologica. 2004;89(2):174-182.

53. Wu W, Shu X, Hovsepyan H, Mosteller RD, Broek D. VEGF receptor expression and signaling in human bladder tumors. Oncogene. 2003;22:3361-3370.

54. Tanaka Y, Abe M, Hiasa M, et al. Myeloma cell-osteoclast interaction enhances angiogenesis together with bone resorption: a role for vascular endothelial cell growth factor and osteopontin. Clin Cancer Res. 2007;13(3):816-823.

55. Zannettino AC, Farrugia AN, Kortesidis A, et al. Elevated serum levels of stromal-derived factor-1 alpha are associated with increased osteoclast activity and osteolytic bone disease in multiple myeloma patients. Cancer Res. 2005;65(5): 1700-1709.

56. Roodman GD. Novel targets for myeloma bone disease. Expert Opin Ther Targets. 2008;12(11):1377-1387.

57. Anderson KC, Shaughnessy JD Jr, Barlogie B, Harousseau JL, Roodman GD. Multiple myeloma. Hematology Am Soc Hematol Educ Program. 2002;1:214-240.

58. Bataille R, Chappard D, Marcelli C, et al. Recruitment of new osteoblasts and osteoclasts is the earliest critical event in the pathogenesis of human multiple myeloma. J Clin Invest. 1991;88(1):62-66.

59. Albers J, Keller J, Baranowsky A, et al. Canonical Wnt signaling inhibits osteoclastogenesis independent of osteoprotegerin. J Cell Biol. 2013;200(4):537-549.

60. Anastas JN, Moon RT. Wnt signalling pathways as therapeutic targets in cancer. Nat Rev Cancer. 2013;13(1):11-26.

61. Katoh Y, Katoh M. Identification and characterization of rat Wnt6 and Wnt10a genes in silico. Int J Mol Med. 2005;15(3):527-531.

62. van Amerongen R, Bowman AN, Nusse R. Developmental stage and time dictate the fate of wnt/beta-catenin-responsive stem cells in the mammary gland. Cell Stem Cell. 2012;11(3):387-400.

63. Wan Y, Lu C, Cao J, et al. Osteoblastic Wnts differentially regulate bone remodeling and the maintenance of bone marrow mesenchymal stem cells. Bone. 2013; 55(1):258-267.

64. Bartis D, Csongei V, Weich A, et al. Down-regulation of canonical and up-regulation of non-canonical Wnt signalling in the carcinogenic process of squamous cell lung carcinoma. PLoS One. 2013;8(3):e57393.

65. Gong Y, Slee RB, Fukai N, et al. LDL receptor-related protein 5 (LRP5) affects bone accrual and eye development. Cell. 2001;107(4):513-523.

66. Boyden LM, Mao J, Belsky J, et al. High bone density due to a mutation in LDLreceptor-related protein 5. N Engl J Med. 2002;346(20):1513-1521.

67. Diarra D, Stolina M, Polzer K, et al. Dickkopf- 1 is a master regulator of joint remodeling. Nature Medicine. 2007;13:156-163.

68. Qiang YW, Chen Y, Stephens O, et al. Myeloma-derived dickkopf-1 disrupts wnt-regulated osteoprotegerin and RANKL production by osteoblasts: a potential mechanism underlying osteolytic bone lesions in multiple myeloma. Blood. 2008;112(1):196-207.

69. Tian E, Zhan F, Walker R, et al. The role of the Wnt-signaling antagonist DKK1 in the development of osteolytic lesions in multiple myeloma. NEnglJMed.2003; 349(26):2483-2494.

70. Gunn WG, Conley A, Deininger L, Olson SD, Prockop DJ, Gregory CA. A crosstalk between myeloma cells and marrow stromal cells stimulates production of DKK1 and interleukin-6: a potential role in the development of lytic bone disease and tumor progression in multiple myeloma. Stem Cells. 2006;24(4): 986-991.

71. Yaccoby S, Ling W, Zhan F, Walker R, Barlogie B, Shaughnessy JD Jr. Antibody-based inhibition of DKK1 suppresses tumor-induced bone resorption and multiple myeloma growth in vivo. Blood. 2007;109(5):2106-2111.

72. Fulciniti M, Tassone P, Hideshima T, et al. Anti-DKK1 mAb (BHQ880) as a potential therapeutic agent for multiple myeloma. Blood. 2009;114(2): 371-379.

73. Oshima T, Abe M, Asano J, et al. Myeloma cells suppress bone formation by secreting a soluble Wnt inhibitor, sFRP-2. Blood. 2005;106(9):3160-3165.

74. Ehrlich LA, Chung HY, Ghobrial I, et al. IL-3 is a potential inhibitor of osteoblast differentiation in multiple myeloma. Blood. 2005;106(4):1407-1414.

75. Giuliani N, Colla S, Morandi F, et al. Myeloma cells block RUNX2/CBFA1 activity in human bone marrow osteoblast progenitors and inhibit osteoblast formation and differentiation. Blood. 2005;106(7):2472-2483.

76. Silvestris F, Cafforio P, De Matteo M, Calvani N, Frassanito MA, Dammacco F Negative regulation of the osteoblast function in multiple myeloma through the repressor gene E4BP4 activated by malignant plasma cells. Clin Cancer Res. 2008;14(19):6081-6091.

77. Prince M, Banerjee C, Javed A, et al. Expression and regulation of Runx2/Cbfa1 and osteoblast phenotypic markers during the growth and differentiation of human osteoblasts. J Cell Biochem. 2001;80(3):424-440.

78. Giuliani N, Rizzoli V. Myeloma cells and bone marrow osteoblast interactions: role in the development of osteolytic lesions in multiple myeloma. Leuk Lymphoma. 2007;48(12):2323-2329.

79. Takeuchi K, Abe M, Hiasa M, et al. Tgf-beta inhibition restores terminal osteoblast differentiation to suppress myeloma growth. PLoS One. 2010;5(3):e9870. 
80. Cocks K, Cohen D, Wisløff F, et al. An international field study of the reliability and validity of a disease-specific questionnaire module (the QLQ-MY20) in assessing the quality of life of patients with multiple myeloma. Eur J Cancer. 2007;43(11):1670-1678.

81. Dunford JE, Thompson K, Coxon FP, et al. Structure-activity relationships for inhibition of farnesyl diphosphate synthase in vitro and inhibition of bone resorption in vivo by nitrogen-containing bisphosphonates. J Pharmacol Exp Ther. 2001;296(2):235-242.

82. Snowden JA, Ahmedzai SH, Ashcroft J, et al. Guidelines for the diagnosis and management of multiple myeloma 2011. Br J Haematol. 2011;154(1):32-75.

83. Brincker H, Westin J, Abildgaard N, et al. Failure of oral pamidronate to reduce skeletal morbidity in multiple myeloma: a double-blind placebo-controlled trial. Br J Haematol. 1998;101(2):280-286.

84. Morgan GJ, Davies FE, Gregory WM, et al. First-line treatment with zoledronic acid as compared with clodronic acid in multiple myeloma (MRC myeloma IX): a randomised controlled trial. Lancet. 2010;376(9757):1989-1999.

85. Drake MT, Clarke BL, Khosla S. Bisphosphonates: mechanism of action and role in clinical practice. Mayo Clin Proc. 2008;83(9):1032-1045.

86. Durie BG. Use of bisphosphonates in multiple myeloma: IMWG response to mayo clinic consensus statement. Mayo Clin Proc. 2007;82(4):516-517; author reply $517-518$.

87. Van den Wyngaert T, Huizing MT, Vermorken JB. Osteonecrosis of the jaw related to the use of bisphosphonates. Curr Opin Oncol. 2007;19(4):315-322.

88. Attal M, Harousseau JL, Leyvraz S, et al. Maintenance therapy with thalidomide improves survival in patients with multiple myeloma. Blood. 2006;108(10): 3289-3294.

89. International Myeloma Working Group. Criteria for the classification of monoclonal gammopathies, multiple myeloma and related disorders: a report of the international myeloma working group. Br J Haematol. 2003;121(5):749-757.

90. Serlin RC, Mendoza TR, Nakamura Y, Edwards KR, Cleeland CS. When is cancer pain mild, moderate or severe? Grading pain severity by its interference with function. Pain. 1995;61(2):277-284.

91. Siemionow K, Lieberman IH. Surgical approaches to metastatic spine disease. Curr Opin Support Palliat Care. 2008;2(3):192-196.

92. Leigh BR, Kurtts TA, Mack CF, Matzner MB, Shimm DS. Radiation therapy for the palliation of multiple myeloma. Int J Radiat Oncol Biol Phys. 1993; 25(5):801-804.

93. Dudeney S, Lieberman IH, Reinhardt M, Hussein M. Kyphoplasty in the treatment of osteolytic vertebral compression fractures as a result of multiple myeloma. J Clin Oncol. 2002;20(9):2382-2387.

94. Garland P, Gishen P, Rahemtulla A. Percutaneous vertebroplasty to treat painful myelomatous vertebral deposits-long-term efficacy outcomes. Ann Hematol. 2011;90(1):95-100.

95. Body J, Facon T, Coleman RE, et al. A study of the biological receptor activator of nuclear factor-kappaB ligand inhibitor, denosumab, in patients with multiple myeloma or bone metastases from breast cancer. Clin Cancer Res. 2006;12(4): $1221-1228$

96. Henry DH, Costa L, Goldwasser F, et al. Randomized, double-blind study of denosumab versus zoledronic acid in the treatment of bone metastases in patients with advanced cancer (excluding breast and prostate cancer) or multiple myeloma. J Clin Oncol. 2011;29(9):1125-1132.

97. Garrett IR, Chen D, Gutierrez G, et al. Selective inhibitors of the osteoblast proteasome stimulate bone formation in vivo and in vitro. J Clin Invest. 2003; 111(11):1771-1782.
98. Heider U, Kaiser M, Müller C, et al. Bortezomib increases osteoblast activity in myeloma patients irrespective of response to treatment. Eur J Haematol. 2006; 77(3):233-238

99. von Metzler I, Krebbel H, Hecht M, et al. Bortezomib inhibits human osteoclastogenesis. Leukemia. 2007;21(9):2025-2034.

100. Hongming H, Jian H. Bortezomib inhibits maturation and function of osteoclasts from PBMCs of patients with multiple myeloma by downregulating TRAF6. Leuk Res. 2009;33(1):115-122.

101. Quach H, Ritchie D, Stewart AK, et al. Mechanism of action of immunomodulatory drugs (IMiDS) in multiple myeloma. Leukemia. 2010;24(1):22-32.

102. Davies FE, Raje N, Hideshima T, et al. Thalidomide and immunomodulatory derivatives augment natural killer cell cytotoxicity in multiple myeloma. Blood. 2001;98(1):210-216.

103. Munshi NC, Anderson KC. New strategies in the treatment of multiple myeloma. Clin Cancer Res. 2013;19(13):3337-3344.

104. Anderson G, Gries M, Kurihara N, et al. Thalidomide derivative CC-4047 inhibits osteoclast formation by down-regulation of PU.1. Blood. 2006;107(8): 3098-3105.

105. Terpos E, Mihou D, Szydlo R, et al. The combination of intermediate doses of thalidomide with dexamethasone is an effective treatment for patients with refractory/relapsed multiple myeloma and normalizes abnormal bone remodeling, through the reduction of sRANKL/osteoprotegerin ratio. Leukemia. 2005;19(11):1969-1976.

106. Terpos E, Christoulas D, Kastritis E, et al. The combination of lenalidomide and dexamethasone reduces bone resorption in responding patients with relapsed/ refractory multiple myeloma but has no effect on bone formation: final results on 205 patients of the Greek myeloma study group. Am J Hematol. 2014;89(1):34-40.

107. Gavriatopoulou M, Dimopoulos MA, Christoulas D, et al. Dickkopf-1: a suitable target for the management of myeloma bone disease. Expert Opin Ther Targets. 2009;13(7):839-848.

108. Body J, Greipp P, Coleman RE, et al. A phase I study of AMGN-0007, a recombinant osteoprotegerin construct, in patients with multiple myeloma or breast carcinoma related bone metastases. Cancer. 2003;97(S3):887-892.

109. Aoki Y, Isselbacher KJ, Pillai S. Bruton tyrosine kinase is tyrosine phosphorylated and activated in pre-B lymphocytes and receptor-ligated B cells. Proc Natl Acad Sci U S A. 1994;91(22):10606-10609.

110. Akinleye A, Furqan M, Adekunle O. Ibrutinib and indolent B-cell lymphomas. Clin Lymphoma Myeloma Leuk. 2013. DOI:10.1016/j.clm1.2013.11.005 (in press).

111. Jain N, O'Brien S. Ibrutinib (PCI-32765) in chronic lymphocytic leukemia. Hematol Oncol Clin North Am. 2013;27(4):851-860.

112. Cinar M, Hamedani F, Mo Z, Cinar B, Amin HM, Alkan S. Bruton tyrosine kinase is commonly overexpressed in mantle cell lymphoma and its attenuation by ibrutinib induces apoptosis. Leuk Res. 2013;37(10):1271-1277.

113. Tai YT, Chang BY, Kong SY, et al. Bruton tyrosine kinase inhibition is a novel therapeutic strategy targeting tumor in the bone marrow microenvironment in multiple myeloma. Blood. 2012;120(9):1877-1887.

114. Bam R, Ling W, Khan S, et al. Role of bruton's tyrosine kinase in myeloma cell migration and induction of bone disease. Am J Hematol. 2013;88(6):463-471.

115. Shinohara M, Koga T, Okamoto K, et al. Tyrosine kinases btk and tec regulate osteoclast differentiation by linking RANK and ITAM signals. Cell.2008;132(5): 794-806. 